\title{
Über das Verhalten der Ideale des Grundkörpers im Klassenkörper.
}

Von Ph. Furtwängler in Wien.

Einleitung. Von dem großen Satze, den Herr D. Hilbert $\left.{ }^{2}\right)$ über den Klassenkörper aufgestellt hat, sind alle Teile vollständig bewiesen bis auf ein e Aussage: daB im Klassenkörper alle Ideale des Grundkörpers Hauptideale, d. h. Körperzahlen werden. Diese Tatsache ist von Herrn Hilbert im Falle der Klassenzahl $h=2$, vom Verfasser allgemeiner für alle Körper mit zyklischer Klassengruppe bewiesen. ${ }^{2}$ ) In der folgenden Arbeit soll der nächst allgemeinere Fall erledigt werden, dab die Klassenzahl $h=l^{2}$ ist und die Klassengruppe $\mathrm{z}$ wei Basisklassen besitzt; $l$ kann dabei gleich 2 sein oder eine ungerade Primzahl bedeuten. ${ }^{3}$ )

Im $\S 1$ wird zunächst allgemein gezeigt, daß, wenn der Grundkörper die Klassenzahl $h=2^{2}=4$ hat, alle seine Ideale im zugehörigen Klassenkörper wirkliche Zahlen werden. In den nächsten Paragraphen wird die Konstitution der Klassengruppe des Klassenkörpers und seiner Unterkörper im angegebenen Falle näher untersucht und insbesondere an zwei numerischen Beispielen gezeigt, dafi die Klassenzahl des Klassenkörpers für unseren Fall sowohl ungerade als gerade sein kann. Danach sind einige Angaben von Herrn Hilber t uber den Fall $h=4$ zu modifizieren. ${ }^{4}$ ) Im letzten Paragraphen endlich wird der Fall $h=l^{2}$, wenn $l$ eine ungerade Primzahl bedeutet, allgemein erledigt.

\section{$\S 1$.}

Der Grundkörper 7 habe das Klassensystem

$$
c_{1}^{x_{1}} c_{2}^{x_{2}}(x=0,1) \text {. }
$$

1) D. Hilbert, Über die Theorie der relativ Abelschen Zahlkörper. Gött. Nachr. 1898, p. 378, Satz 10.

2) Pb. Furtwängler, Allgemeiner Existenzboweis usw., Math. Ann. 63 (1906), p. 1 ( 88$)$.

3) Im Falle $l=2$ wird angenommen, daß dis Klassenzahl im weiteren und engeren Sinne gleich 4 sei. Gedruckte).

4) D. Hilbert, 1. c. Satz $16 b$ und Satz $16 c$ (von letztem Satz das kursiv 
Es existieren dann in $k$ zwei singuläre Primärzahlen $\omega_{1}, \omega_{2}$, die resp. zu den Untergruppen $c_{1}^{x_{1}}$ und $c_{2}^{x_{2}}$ gehören, so daß der Klassenkörper von $k$ durch:

$$
K k=\left(k, \sqrt{\omega_{1}}, \sqrt{\omega_{2}}\right)
$$

gegeben ist. Betrachten wir zunächst den Unterkörper des Ǩlassenkörpers $K_{1}=\left(k, \sqrt{\omega_{1}}\right)$, so können wir, wie aus der allgemeinen Theorie des Klassenkörpers $\left.{ }^{1}\right)$ folgt, sein Klassensystem durch

$$
C^{F(S)}
$$

darstellen; $C$ bedeutet dabei eine Klasse aus $K_{1}$, deren Relativnorm in $k$ in $c_{1}$ fällt, und $S$ ist die Substitution $\sqrt{\omega_{1}} \mid-\sqrt{\omega_{1}}$. Der Körper $K_{1}$ besitzt nun sicher einen ambigen Komplex, der entweder eine ambige Klasse enthalten kann oder nicht.

Enthält er eine ambige Klasse, so folgt aus der allgemeinen Theorie, daß $z w e i$ unabhängige Klassenverbände aus $k$ in $K_{1}$ in die Hauptklasse tibergehen, d. $h$. in diesem Falle gehen bereits sämtliche Klassen aus $k$ in $K_{1}$, um so mehr also in $K K$ in die Hauptklasse über.

Enthält der ambige Komplex keine ambige Klasse, so gehen nicht alle Klassen aus $k$ in $K_{1}$ in die Hauptklasse über, sondern es gibt eine Klasse $c$ in $k$, die in $K_{1}$ die Äquivalenz

$$
A^{S-1} \sim c \propto 1
$$

befriedigt, wo $A$ eine Klasse des ambigen Komplexes bedeutet. In diesem Falle gehe ich durch Adjunktion von $\sqrt{\omega_{2}}$ zu $K_{1}$ zum Klassenkörper $K h$ über und zeige, dah bei dieser Adjunktion $c$ in die Hauptklasse übergeht.

Nach der allgemeinen Theorie muß es in $K_{1}$ eine Klasse $C_{0}$ geben, die in $K_{1}$ nicht Hauptklasse ist und in $K k$ in die Hauptklasse übergeht. Mit $C_{0}$ gehen dann offenbar auch alle Klassen

$$
C_{0}^{(S-1)^{n}}=C_{n}
$$

in $K k$ in die Hauptklasse. Da die $(S-1)^{2 \text { to }}$ symbolische Potenz einer Klasse gleichzeitig Quadrat einer Klasse ist, muß für einen gewissen Exponenten $n$

$$
C_{n}^{q_{n}} \sim 1, \quad C_{n-1}^{q_{n}} \chi 1
$$

sein, wo $q_{n}$ eine ungerade Zahl bedeutet. Die Klasse $C_{n-1}$ befriedigt dann in $K_{1}$ die $\ddot{A} q u i v a l e n z:$

$$
C_{n-1}^{q_{n}(s-1)} \sim 1, \quad C_{n-1}^{q_{n}} \propto 1 .
$$

Da nach Annahme keine ambige Klasse in $K_{1}$ existieren soll, muß $C_{n-1}^{q_{n}} \sim c$ gelten; es geht also $c$ ebenfalls in $K k$ in die Hauptklasse über.

1) Ph. Furtwängler, l. c. $\$ 6$ und Gött. Nachr. 1907, § 4. 


\section{$\$ 2$.}

In diesem und dem folgenden Paragraphen soll die Konstitution der Klassengruppe des Klassenkörpers $K k$ und der unverzweigten Zwischenkörper zwischen Grundkörper und Klassenkörper genauer untersucht werden. Wir wählen zu diesem $Z_{\text {weck }}$ folgende Bezeichnungen. Der Grundkörper sei $k$ mit der Klassengruppe

$$
c_{1}^{x_{1}} c_{2}^{x_{2}}(x=0,1),
$$

wobei wir noch die Klasse $c_{1} c_{2}$ mit $c_{3}$ bezeichnen.

Es existieren dann drei unverzweigte relativquadratische Körper in bezug auf $k$ :

$$
\begin{aligned}
& K_{1}=\left(k, \sqrt{\omega_{1}}\right) \text { zur Klassengruppe 1, } c_{1} \text { gehörig, } \\
& K_{2}=\left(k, \sqrt{\omega_{2}}\right) \quad " \quad \text { " } 1, c_{2} \quad " \\
& K_{3}=\left(k, \sqrt{\omega_{3}}\right) \quad n \quad, \quad 1, c_{3} \quad n \text {. }
\end{aligned}
$$

Dabei sind $\omega_{1}, \omega_{2}, \omega_{3}$ singuläre Primärzahlen in $k$ und $\omega_{3}=\omega_{1} \omega_{2}$. Der Klassenkörper ist durch $\left(k, \sqrt{\omega_{1}}, \sqrt{\omega_{2}}\right)$ bestimmt. Wir bezeichnen endlich folgende Substitutionen:

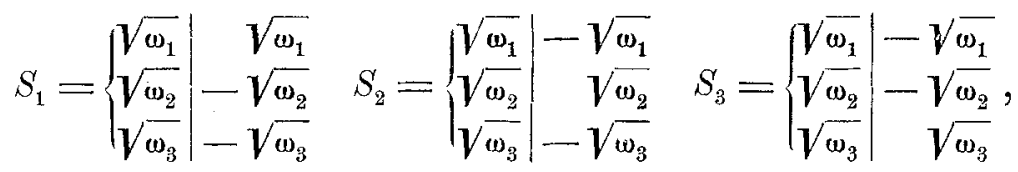

zwischen denen die Beziehung: $S_{1} S_{2} S_{3}=1$ besteht. Der Übergang vom Klassenkörper zum Körper $K_{i}$ erfolgt dann durch symbolische Potenzierung mit $1+S_{i}$.

Wir untersuchen zunächst die möglichen Fälle, die die Klassengruppe von $K_{1}$ bieten kann. Das Klassensystem von $K_{1}$ ist darstellbar in der Grestalt:

$$
C_{1}^{F^{\prime}\left(S_{2}\right)}
$$

wo $C_{1}$ eine Klasse bedeutet, deren Relativnorm in $k$ in $c_{1}$ füllt, und wobei ein-für allemal verabredet werden soll, daß als Einheitselement der Klassengruppe das System aller Klassen mit ungeradem Exponenten angenommen wird. Wir haben nun mehrere Fälle zu unterscheiden :

$$
\text { I. } C_{1}^{2} \sim 1 .
$$

a) $C_{1}^{S_{2}-1} \sim 1$. In diesem Falle existiert in $K_{1}$ eine ambige Klasse und es gehen daher alle Klassen aus $k$ bereits in $K_{1}$ in die Hauptklasse über. Das Klassensystem von $K_{1}$ ist in diesem Falle:

$$
C_{1}^{x}(x=0,1)
$$


und die Klassenzahl von $K l$ wird ungerade. Aus dem letzten Umstande ergibt sich sofort, daß in unserem Falle auch die Klassenzahlen von $K_{2}$ und $K_{3}$ gleich 2 sein müssen und daß auch in diesen Körpern sämtliche Klassen aus $k$ in die Hauptklasse übergehen.

b) $C_{1}^{S_{2}-1} \nsim 1$. Es existiert jettt keine ambige Klasse in $K_{1}$ und daher geht jetzt nur eine Klasse aus $k$ in $K_{1}$ in die Hauptklasse über; es gilt nämlich:

$$
c_{1} \propto C_{1}^{S_{2}+1} \sim C_{1}^{S_{2}-1} \nsim 1
$$

Das Klassensystem in $K_{1}$ bildet jetzt keine zyklische, sondern eine Abelsche Gruppe mit zwei Basisklassen:

$$
C_{1}^{x} c_{1}^{y}(x, y=0,1) \text {. }
$$

Die Klassenzahl von $K k$ ist eine gerade Zabl.

$$
\text { II. } C_{1}^{2} \nsim 1 \text {. }
$$

Da die Relativnorm von $C_{1}^{2}$ in $k$ in die Hauptklasse fällt, $\operatorname{muß} C_{1}^{2} \operatorname{der}\left(S_{2}-1\right)^{\text {ten }}$ Potenz einer Klasse äquivalent sein, d. b. es muls eine Aquivalenz gelten:

$$
C_{1}^{2} \sim C_{1}^{m\left(S_{2}-1\right)},
$$

wo $m$ eine ganze Zahl bedeutet. Die Zahl $m$ muß ungerade sein; denn wäre $m=2 m_{1}$, so würde folgen:

$$
C_{1}^{2} \propto C_{1}^{2 m_{1}\left(S_{2}-1\right)} \sim C_{1}^{2 m_{1}{ }^{2}\left(S_{2}-1\right)^{2}} \sim C_{1}^{2 m_{1}{ }^{3}\left(S_{2}-1\right)^{3}} \ldots \propto 1,
$$

was nach Annahme ausgeschlossen ist. Ist nun:

$$
C_{1}^{2^{n}} \sim 1
$$

so folgt aus der vorletzten Äquivalenz:

$$
C_{1}^{S_{2}-1} \sim C_{1}^{2 m^{\prime}}
$$

wenn man $m^{\prime}$ aus der Kongruenz:

$$
m m^{\prime} \equiv 1\left(2^{n}\right)
$$

bestimmt.

Es liegt also in unserem Falle $C_{1}^{S_{2}-1}$ bereits im Hauptverbande und daher kann nur ein unabhängiger Klassenverband in $K_{1}$ existieren; das Klassensystem von $K_{1}$ wird

$$
C_{1}^{x}\left(x=0,1, \ldots 2^{n}-1\right) \quad(n>1) .
$$

Die Klassenzahl von $K k$ ist wiederum gerade. 


\section{\$ 3.}

Es soll jetzt untersucht werden, in welcher Kombination die Fälle $\mathrm{I} a, \mathrm{I} b$, II des vorigen Paragraphen bei den Körpern $K_{1}$, $K_{2}, K_{3}$ auftreten können.

Wenn der Fall I $a$ bei einem der drei Körper eintritt, so tritt er bei allen dreien auf, wie ohne weiteres aus der Tatsache folgt, daß die Klassenzahl von $K K$ in diesem Falle ungerade ist.

Wir können daher jetzt annehmen, daß der Fall I $a$ bei keinem der drei Körper $K_{i}$ eintritt, und wollen zunächst zeigen, daß die Klassengruppe von $K k$ in jedem Falle zyklisch ist. Liegt in einem der Körper $K_{i}$ der Fall II vor, so ist seine Klassengruppe zyklisch und bleibt es daher auch beim Übergang zu $K k$, wie aus der allgemeinen Theorie des Klassenkörpers folgt. Wir haben also nur noeh den Fall zu erwägen, daß bei allen drei Körpern $K_{i}$ der Fall Ib eintritt (was nicht möglich ist, wie sich später zeigen wird). Beim Übergang von $K_{i}$ zu $K k$ könnte dann wieder der Fall Ib vorliegen und so ein Klassensystem

$$
D^{x} E^{y}(x, y=0,1)
$$

in $K k$ liefern. Es dürfte dann kein ambiger Komplex mit ambiger Klasse in $K \eta_{6}$ bezüglich $K_{1}, K_{2}, K_{3}$ existieren. Daraus würde folgen, dab alle Klassen:

$$
1, D, S_{1} D, S_{2} D, S_{3} D
$$

verschieden sein müßten, was bei unseren Annahmen ausgeschlossen ist, da nur vier verschiedene Klassen in $K \hbar$ existieren. Daraus folgt aber, daßs die Klassengruppe von $K k$ zyklisch sein mul, da eine andere Annahme zu einem Widerspruch führt.

Es existiert also e in unverzweigter relativquadratischer Körper in bezug auf $K k$ :

$$
K^{\prime}=(K k, \sqrt{\Omega})
$$

der nach der allgemeinen Theorie nicht relativ Abelsch in bezug auf $k$ sein kann. ${ }^{1}$ ) Dagegen ist er ein relativ Galoisscher Körper bezüglich $k$; denn da nur e in e Art singulärer Primärzahlen in $K k$ existieren kann und $S_{1} \Omega, S_{2} \Omega, S_{3} \Omega$ zugleich mit $\Omega$ singuläre Primärzahlen sind, mulo gelten:

$$
S_{i} \Omega=A_{i}^{2} \Omega \quad(i=1,2,3),
$$

wo die Zahlen $A_{i}$ dem Körper $K k$ angehören. Durch Bildung der Relativnorm in bezug auf $K_{i}$ folgt:

$$
A_{i}^{1+S_{i}}=\varepsilon_{i}, \varepsilon_{i}= \pm 1 \quad(i=1,2,3) .
$$

1) Ph. Furtwängler, Eine chirakteristische Eigenschaft des Klassenkörpers, Gött. Nachr. 1906 und 1907. 
Ich will nachweisen, daß

$$
\varepsilon_{1} \cdot \varepsilon_{2} \cdot \varepsilon_{3}=-1
$$

sein muß. Sind alle drei Zahlen $\varepsilon_{i}=-1$, so ist die Beziehung erfüllt; $\theta$ s sei also etwa $\varepsilon_{1}=+1$.

Da jetzt $A_{1}^{1+S_{1}}=1$, ist $A_{1}=B_{1}^{1-S_{1}}$, wo $B_{1}$ eine Zahl aus $K k$ ist. Es gilt also:

$$
S_{1}\left(\Omega B_{1}^{2}\right)=\Omega B_{1}^{2}
$$

d. h. $\Omega B_{1}^{2}$ ist eine Zahl aus $K_{1}$. Da man $\Omega B_{1}^{2}$ an Stelle von $\Omega$ setzen kann, kann man also auch $\Omega$ als Zahl aus $K_{1}$ wählen. In diesem Falle ist dann die Klassengruppe von $K_{1}$ nicht zyklisch, sondern Abelsch mit zwei Basisklassen, da in $K_{1}$ zwei unabhängige singuläre Primärzahlen $\omega_{2}, \Omega$ existieren. Umgekehrt gilt auch, daß $\varepsilon_{1}=+1$ sein muß, wenn man $\Omega$ als $\mathrm{Zahl}$ aus $K_{1}$ wählen kann, denn es ist dann $A_{1}=1$.

Es sei also $\Omega$ jetzt eine Zahl aus $K_{1}$, daher $A_{1}=1$; wir haben nachzuweisen, daß dann $\varepsilon_{2}=-\varepsilon_{3}$ ist. Es gilt jetzt:

$$
S_{1} \Omega=\Omega, \quad S_{2} \Omega=A_{2}^{2} \Omega, \quad S_{3} \Omega=A_{3}^{2} \Omega \text {. }
$$

Daraus folgt:

$$
\begin{aligned}
& S_{3} \Omega=S_{1} S_{2} \Omega=S_{1} A_{2}^{2} \Omega \\
& S_{3} \Omega=S_{2} S_{1} \Omega=A_{2}^{2} \Omega .
\end{aligned}
$$

Damit der Körper $K^{\prime}$ kein Abelscher in bezug auf $k$ sei, mulo gelten:

$$
A_{2}=-S_{1} A_{2}=A_{3} \text {. }
$$

Es wird also:

$$
\varepsilon_{3}=A_{3}^{1+S_{3}}=A_{2}^{1+S_{1} S_{2}}=A_{2} \cdot\left(-S_{2} A_{2}\right)=-A_{2}^{1+S_{2}}=-\varepsilon_{2} .
$$

Es ist also in jedem Falle:

$$
\varepsilon_{1} \cdot \varepsilon_{2} \cdot \varepsilon_{3}=-1
$$

und, abgesehen von Indizesvertauschungen, ergeben sich daher nur die beiden Möglichkeiten:

$$
\begin{aligned}
& \varepsilon_{1}=-1, \quad \varepsilon_{2}=-1, \quad \varepsilon_{3}=-1 \\
& \varepsilon_{1}=+1, \quad \varepsilon_{2}=+1, \quad \varepsilon_{3}=-1 .
\end{aligned}
$$

Nach dem, was oben ausgeführt ist, heißt das aber auf die Körper $K_{i}$ übertragen, daß entweder bei allen drei Körpern der Fall II des vorigen Paragraphen eintreten muß oder nur bei einem. 
Aus unseren bisherigen Ausführungen ergeben sich demnach folgende drei Typen :

Typus $A$ : Klassenzahl von $K h$ ungerade.

Klassensysteme von:

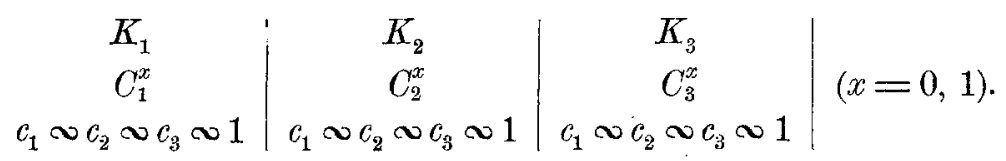

Typus $B$ : Klassenzahl von $K k$ gleich 2 ; alle drei Körper $K_{i}$ haben zyklische Klassengruppen:

Klassensysteme von:

$$
\begin{aligned}
& \begin{array}{c|c|c|}
K_{1} & K_{2} & K_{3} \\
C_{1}^{x} & C_{2}^{x} & C_{3}^{x} \\
c_{1} \sim 1, c_{2} \sim c_{3} \nsucc 1 & c_{2} \sim 1, c_{1} \sim c_{3} \psi 1 & c_{3} \sim 1, c_{1} \sim c_{2} \nsim 1
\end{array} \\
& (x=0,1,2,3) \text {. }
\end{aligned}
$$

Zu diesem Schema sind noch einige Begründungen zu geben. Zunächst ist klar, daß die Klassenzahl in allen drei Körpern $K_{i}$ dieselbe sein mul, da sie das Doppelte der Klassenzahl von $K k$ ist; sie sei etwa gleich $2^{n}$. Der Klassenkörper $K^{2} k$ von $K k$ ist dann gleichzeitig Klassenkörper von $K_{1}, K_{2}, K_{3}$ und vom Relativgrad $2^{n}$ bezüglich dieser. Ist nun $\mathfrak{p}_{1}$ ein Primideal aus $c_{1}$, so zerfällt dies in $K_{1}$ in zwei verschiedene Faktoren, die dann beim Übergang nach $K^{2} k$ nicht mehr zerlegt werden. Es zerfallen also alle Primideale aus $c_{1}$ in $K^{2} k$ in zwei verschiedene Primfaktoren. Betrachten wir anderseits den Körper $K_{2}$, so bleibt $\mathfrak{p}_{1}$ in ihm Primideal und geht entweder in die Hauptlzlasse oder in eine Klasse, deren Quadrat in die Hauptklasse fällt, über. Bei dem Übergang zum Körper $K^{2} k$ zerfällt dann nach dem Zerlegungssatz für den Klassenkörper $\left.{ }^{1}\right) \mathfrak{p}_{1}$ in $2^{n}$ oder $2^{n-1}$ verschiedene Primfaktoren. Da diese Zahl 2 sein mub und $n>1$ ist, kann der erste Fall überhaupt nicht eintreten, sondern es mub der zweite Fall stattinden mit $n=2$. Es gilt dann also in $K_{2}: c_{1} \sim C_{2}^{2} \nsim 1$.

Indem man dieselben Überlegungen für alle übrigen Klassen und Körper durchführt, kommt man zur Begründung aller Angaben des Schemas. 
Typus $C:$ Klassenzahl von $K k$ gleich $2^{n}(n>0)$; nur Körper $K_{3}$ hat zyklische Klassengruppe.

Klassensysteme ron:

$$
\begin{aligned}
& \begin{array}{c|c|c}
K_{1} & K_{2} & K_{3} \\
C_{1}^{x} c_{1}^{y} & C_{2}^{x} c_{2}^{y} & C_{3}^{z} \\
c_{2} \propto 1, c_{1} \propto c_{3} \nsucc 1 & c_{1} \sim 1, c_{2} \propto c_{3} \nsucc 1 & -
\end{array} \\
& \left(x, y=0,1 \quad z=0,1, \ldots, 2^{n+1}-1\right) \text {. }
\end{aligned}
$$

Zur Begründung des Schemas sei noch folgendes bemerkt: Der Klassenkörper $K^{2} 7_{6}$ von $K /$ ist auch Klassenkörper für $K_{3}$; in ihm werden daher die Primideale aus $c_{3}$ in zwei verschiedene Faktoren zerlegt. Daraus folgt, daß $c_{3}$ in $K_{1}$ nicht in die Hauptklasse übergehen kann, weil sonst die Primideale aus $c_{3}$ in $\hbar^{2} \hbar_{k}$ in mehr als zwei Primfaktoren zerfallen würden. Da auch $c_{1} \propto 1$ in $K_{1}$, muß $c_{2} \sim 1$ in $K_{1}$ sein. Entsprechendes gilt für $K_{2}$.

Für den speziellen Fall $n=1$ lassen sich auch über den Körper $K_{3}$ noch genauere Angaben machen. Wir fügen daher noch folgrendes spezielle Schema hinzu:

$$
\begin{aligned}
& \text { Typus } C, n=1 \text { : Klassenzahl von } K k \text { gleich } 2 \text {. } \\
& \text { Klassensysteme } \nabla \text { on: } \\
& \begin{array}{c|c|c}
K_{1} & K_{2} & K_{3} \\
C_{1}^{x} c_{1}^{y y} & C_{2}^{x} c_{2}^{y} & C_{3}^{z} \\
c_{2} \sim 1, c_{1} \sim c_{3} \psi 1 & c_{1} \sim 1, c_{2} \sim c_{3} \propto 1 & c_{1} \sim c_{2} \sim c_{3} \sim 1
\end{array} \\
& (x, y=0,1 \quad z=0,1,2,3) \text {. }
\end{aligned}
$$

Der Klassenkörper von $K_{1}$ repräsentiert jetzt den Typus $A$ und daher wird ein Primideal aus $a_{1}$ in $K_{1}$ in zwei Faktoren zerfallen, von denen jeder in $K^{2} k$ nochmals in zwei Faktoren zerfällt; die Primideale aus $c_{1}$ zerfallen also in $K^{2} k$ in vier verschiedene Primfaktoren. Aus diesem Grande mub in $K_{3}: c_{1} \sim 1$ gelten. In entsprechender Weise schließt man mit Hilfe des Körpers $K_{2}$, daß auch $c_{2} \sim 1$ in $K_{3}$ ist.

Daß die hier aufgestellten Typen in Wirklichkeit alle vorkommen, ergeben die bisherigen Ausführungen nicht. Dies soll in den nächsten Paragraphen dadurch gezeigt werden, daß für die Typen $A, B, C$ je ein Beispiel gegeben wird.")

1) Es ist auch gruppentbeoretisch leicht zn zeigen, dab die beiden einzigen nicht kommutativen Gruppen von der Ordnung 8 diejenigen sind, die der Relativgruppe von $K^{2} k$ bei dem Typus $B$ und $C$ mit $n=1$ entsprechen; Typus $R$ entspricht der Hamiltonschen Quaternionengruppe. 
Wir notieren schließlich noch folgenden Satz, dessen Richtigkeit aus dem Vorstehenden folgt:

Satz: Konstruiert man zu einem Körper $k$, dessen Klassenzahl genau dureh 4 teilbar ist, den Klassenkörper $K k$ und zu diesem wieder den Klassenkörper $K^{2} k$, so ist die Klassenzahl von $K^{2} \eta_{\text {ungerade und }} K^{2} k$ ist ein relativGaloisseher Körper in bezug auf $k$. (Die Konstruktion der Klassenkörper bezieht sich dabei immer nur auf die Relativgrade, die eine Potenz von 2 sind.)

\section{$\$ 4$.}

Wir geben zunächst ein Beispiel zu dem Typus $A$; dies liefert der Körper $(V-21)$ mit der Diskriminante -84 . Wir bestimmen zunächst seine Klassengruppe, indem wir die Zerlegung der Zahlen 2, 3, 5, 7 untersuchen $(\sqrt{84}<10)$.

Ihre Zerlegung in Primideale ist die folgende:

$$
\begin{aligned}
& 2=p_{2}^{2} \quad p_{2}=(2,1+\sqrt{-21}) \\
& 3=p_{3}^{2} \quad p_{3}=(3, \sqrt{-21}) \\
& 7=\mathfrak{p}_{7}^{2} \quad \mathfrak{p}_{7}=(7, \sqrt{-21}) \quad \mathfrak{p}_{3} \mathfrak{p}_{7} \sim 1 \\
& 5=p_{5} \cdot \bar{p}_{5} \quad \mathfrak{p}_{5}=(5,2+\sqrt{-21}) \quad p_{5}^{2}=2+\sqrt{-21} \backsim 1 \text {. }
\end{aligned}
$$

Aus

$$
30=2.3 .5=(3+\sqrt{-21})(3-\sqrt{-21})
$$

folgt

$$
\mathfrak{p}_{2} \mathfrak{p}_{3} \sim \mathfrak{p}_{5} \propto 1
$$

denn $\mathfrak{p}_{3}$ kann nicht $\sim 1$ sein, weil \pm 5 nicht Norm einer Körperzahl aus $(\sqrt{-21})$ ist. Da $\mathfrak{p}_{2}, \mathfrak{p}_{3}, \mathfrak{p}_{5}$ nicht äquivalente Ideale sind, die nicht in der Hauptklasse liegen, ist die Klassenzahl von $(\sqrt{-21})$ gleich 4 und die Klassengruppe:

$$
c_{1}^{x_{1}} c_{2}^{x_{2}}(x=0,1),
$$

wobei die Bezeichnung der Klassen so gewählt sei, daß in

liegt.

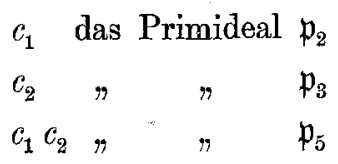


Der Klassenkörper von $(\sqrt{-21})$ wird:

$$
K k=(\sqrt{-21}, \sqrt{-3}, \sqrt{-7})=(i, \rho, \sqrt{7}),
$$

wenn wir unter $\rho$ eine primitive dritte Einheitswurzel verstehen.

Die Klassenzahl von $K k$ ist, wie wir jetzt zeigen wollen, ungerade und daher liegt Typus $A$ vor.

Die Klassenzahl von (i) ist gleich $1 ; 3$ ist primäres Primideal im Körper (i) und $-3 \equiv 1(4)$ eine zugehörige Primärzahl; daher ist die Klassenzahl von $(i, \rho)$ ungerade. $\left.{ }^{1}\right)$

Die Zahl 7 zerfällt in $(i, \rho)$ in zwei nichtprimäre Primfaktoren:

$$
7=(2+\sqrt{-3})(2-\sqrt{-3}) .
$$

Daß $2+\sqrt{-3}$ nicht primär ist, folgt, weil für die Einheit $i+p$ gilt:

$$
\left(\frac{i+\rho}{2+\sqrt{-3}}\right)_{(i, \varrho)}=\left(\frac{-\rho}{2+\sqrt{-3}}\right)_{(\varrho)}=\left(\frac{-1}{2+\sqrt{-3}}\right)_{(\varrho)}=\left(\frac{-1}{7}\right)=-1 .
$$

Daher ist die Klassenzahl von $(i, \rho, \sqrt{7})$ ungerade. $\left.^{1}\right)$

Daß der Typus $A$ vorliegt, ergibt sich auch daraus, dab alle Ideale aus $(\sqrt{-21})$ bereits in $K_{1}, K_{2}, K_{3}$ Hauptideale werden, wie folgende Angaben zeigen:

Körper $(\sqrt{-21}, \sqrt{-3})=(\sqrt{7}, \sqrt{-3})$;

$$
\begin{aligned}
2 & =(3+\sqrt{7})(3-\sqrt{7}), 3=(\sqrt{-3})(-\sqrt{-3}) \\
" \quad(\sqrt{-21}, \sqrt{-7})=(\sqrt{3}, \sqrt{-7}) & \\
-2 & =(1+\sqrt{3})(1-\sqrt{3}),-3=(\sqrt{3})(-\sqrt{3}) \\
" \quad(\sqrt{-21}, \sqrt{21}) & =(\sqrt{21}, i) ; \\
2 & =(1+i)(1-i),-3=\frac{3+\sqrt{21}}{2} \cdot \frac{3-\sqrt{21}}{2} .
\end{aligned}
$$

\section{$\$ 5$.}

Beispiele für die Typen $B$ und $C$ liefert uns die Untersuchung des Körpers $k(\sqrt{-195})=k(\sqrt{-3.5 .13})$. Wir stellen zunächst die Klassengruppe dieses Körpers fest, indem wir die Zerlegung von $2,3,5,7,11,13$ in $k$ untersuchen.

1) Da die Arzahl der ambigen Komplexe gleich 1 ist; vgl. Pb. Furtwängler, Über die Reziprozitätsgesetze usw,, Math. Ann. 58, p. 43. 
$3,5,13$ gehen in der Diskriminante auf und werden daher in $k$ Quadrate von Primidealen:

$$
3=p_{3}^{2}, \quad 5=p_{5}^{2}, \quad 13=p_{13}^{2}, \quad p_{3} p_{5} p_{13} \sim 1 .
$$

Von den noch übrigen Primzahlen 2, 7, 11 bleibt 2 wegen

$$
-195 \equiv 5(8)
$$

Primzahl, während 7 und 11 zerlegt werden:

$$
\begin{array}{r}
7=\mathfrak{p}_{7} \cdot \overline{\mathfrak{p}}_{7}, \mathfrak{p}_{7}=\left(7, \frac{1+\sqrt{-195}}{2}\right), \mathfrak{p}_{7}^{2}=\frac{1+\sqrt{-195}}{2} \sim 1, \\
11=\mathfrak{p}_{11} \cdot \overline{\mathfrak{p}}_{11}, \mathfrak{p}_{11}=\left(11, \frac{17+\sqrt{-195}}{2}\right), \mathfrak{p}_{11}^{2}=\frac{17+\sqrt{-195}}{2} \sim 1 .
\end{array}
$$

Ferner folgt ans:

$105=3.5 .7=\frac{15+\sqrt{-195}}{2} \cdot \frac{15-\sqrt{-195}}{2}, \quad$ dab $\quad \mathfrak{p}_{3} \mathfrak{p}_{5} \mathfrak{p}_{7} \sim 1$ und aus

$$
55=5.11=\frac{3+\sqrt{-195}}{2} \cdot \frac{3-\sqrt{-195}}{2}, \quad \text { daß } \quad \mathfrak{p}_{11} \mathfrak{p}_{5} \sim 1 .
$$

$\mathrm{Da}$

$$
p_{3} \nsim p_{5} \nsim p_{7} \nsim 1
$$

ist, ergibt sich aus dem Vorstehenden, daß die Klassenzahl von $k$ gleich 4 ist und daß die Klassengruppe in der Gestalt:

$$
c_{1}^{x_{1}} c_{2}^{x_{2}} \quad(x=0,1)
$$

geschrieben werden kann, wobei die Bezeichnung so gewählt sei, dak die Klassen $c_{1}, c_{2}, c_{3}=c_{1} c_{2}$ resp. die Primideale $\mathfrak{p}_{3}, \mathfrak{p}_{7}, \mathfrak{p}_{5}$ enthalten.

Der Klassenkörper $K k$ von $k$ ist:

$$
K k=(\sqrt{-195}, \sqrt{-3}, \sqrt{5})=(\sqrt{-3}, \sqrt{5}, \sqrt{13}) .
$$

Ich behaupte, daß seine Klassenzahl g e rade ist, was man auf verschiedene Weise zeigen kann.

1. Beweis: Es gibt in $K \hbar$ eine singuläre Primärzahl :

$$
-1+2 \sqrt{-3} \equiv 1(4) .
$$

$-1+2 \sqrt{-3}$ ist das Quadrat des Ideals $p=(\sqrt{13},-1+2 \sqrt{-3})$; es ist also nur nachzuweisen, daß $-1+2 \sqrt{-3}$ nicht Quadrat einer 
Zahl aus $K k$ ist. Wir betrachten zu dem Zweck das Primideal $\mathfrak{P}_{5}=\left(\sqrt{5}, \frac{1+\sqrt{-39}}{2}\right)$, dessen Relativnorm im Körper $(\sqrt{-39})$ gleich $\mathfrak{p}_{5}^{\prime}=\left(5, \frac{1+\sqrt{--39}}{2}\right)$ ist. Für dieses gilt:

$$
\begin{gathered}
\left(\frac{-1+2 \sqrt{-3}}{\mathfrak{P}_{5}}\right)_{K k}=\left(\frac{-1+2 \sqrt{-3}}{\mathfrak{p}_{5}^{\prime}}\right)_{(\sqrt{-3}, \sqrt{13})}= \\
=\left(\frac{-1+2 \sqrt{-3}}{5}\right)_{(\sqrt{-3})}=\left(\frac{13}{5}\right)=-1 .
\end{gathered}
$$

Es kann also $-1+2 \sqrt{-3}$ nicht Quadrat einer Zabl aus $K k$ sein, womit der gewünschte Beweis erbracht ist.

2. Beweis: Wir zeigen, daß die Klassenzahl von $(\sqrt{-39})$ gleich 4 und die Klassengruppe zyklisch ist, indem wir die Zerlegung der Zahlen 2, 3, 5 im Körper $(\sqrt{-39})$ prüfen. Es wird:

$$
\begin{array}{lll}
2=\mathfrak{p}_{2}^{\prime} \cdot \overline{\mathfrak{p}}_{2}^{\prime} & \mathfrak{p}_{2}^{\prime}=\left(2, \frac{1+\sqrt{-39}}{2}\right) & \mathfrak{p}_{2}^{\prime 4}=\frac{5+\sqrt{-39}}{2} \sim 1 \\
3=\mathfrak{p}_{3}^{\prime 2} & \mathfrak{p}_{3}^{\prime}=(3, \sqrt{-39}) & \mathfrak{p}_{3}^{\prime 2}=3 \sim 1 \\
5=\mathfrak{p}_{5}^{\prime} \cdot \overline{\mathfrak{p}}_{5}^{\prime} & \mathfrak{p}_{5}^{\prime}=\left(5, \frac{1+\sqrt{-39}}{2}\right) & \mathfrak{p}_{5}^{\prime 4}=1-4 \sqrt{-39} \sim 1 .
\end{array}
$$

Ferner schlielit man aus:

$$
\mathfrak{p}_{2}^{\prime} \cdot \mathfrak{p}_{5}^{\prime}=\frac{1+\sqrt{-39}}{2}, \quad 12=\frac{3+\sqrt{-39}}{2} \cdot \frac{3-\sqrt{-39}}{2},
$$

$\mathrm{da} b$

$$
\mathfrak{p}_{2}^{\prime} \mathfrak{p}_{5}^{\prime} \sim 1, \quad \mathfrak{p}_{3}^{\prime} \mathfrak{p}_{2}^{\prime 2} \sim 1
$$

$\mathrm{Da}$ überdies leicht festzustellen ist, daß nicht bereits $\mathfrak{p}_{2}^{\prime 2} \sim 1$ ist, folgt, daß das Klassensystem von $k^{\prime}(\sqrt{-39})$ in der Gestalt

$$
c^{x} \quad(x=0,1,2,3)
$$

darstellbar ist, wo die Klasse $c$ so gewählt sei, daß $\mathfrak{p}_{5}^{\prime}$ in ihr liegt. $\operatorname{Im}$ Körper $K_{3}=(\sqrt{-39}, \sqrt{5})$ wird dann $\mathfrak{p}_{5}^{\prime}=\mathfrak{P}_{5}^{2}$, wo $\mathfrak{P}_{5}$ die oben angegebene Bedeutung hat. Es ist dann sicher $\mathfrak{P}_{5}^{8} \sim 1$, aber nicht bereits $\mathfrak{B}_{5}^{2} \sim \mathfrak{p}_{5}^{\prime} \sim 1$, denn sonst würde durch Bildung der Relativnorm bezüglich $k^{\prime}(\sqrt{-39})$ folgen, daß in $k^{\prime} \mathfrak{p}_{5}^{\prime 2} \sim 1$ gelten 
müßte, was unmöglich ist. Es existiert also in $K_{3}$ eine Klasse mit dem Exponenten 4 und daher kann die Klassenzahl von

nicht ungerade sein.

$$
K k=\left(K_{3}, \sqrt{13}\right)
$$

Für die Körper $K_{1}, K_{2}, K_{3}$ gilt in unserem Falle folgendes:

\begin{tabular}{|c|c|c|}
\hline K о r p e r & Klassensystem & $\begin{array}{c}\text { Verhalten der Ideale } \\
\text { aus } k\end{array}$ \\
\hline $\begin{array}{l}K_{1}=(\sqrt{-195}, \sqrt{13}) \\
\left(\frac{13}{\mathfrak{p}_{3}}\right)_{k}=\left(\frac{13}{3}\right)=+1\end{array}$ & $C_{1}^{x} c_{1}^{y} \quad(x, y=0,1)$ & $c_{2} \sim 1, c_{1} \sim c_{3} \chi 1$ \\
\hline $\begin{array}{l}K_{2}=(\sqrt{-195}, \sqrt{-3}) \\
\left(\frac{-3}{\mathfrak{p}_{7}}\right)_{k}=\left(\frac{-3}{7}\right)=+1\end{array}$ & $C_{2}^{x} c_{2}^{y} \quad(x, y=0,1)$ & $c_{1} \sim 1, c_{2} \sim c_{3} \chi 1$ \\
\hline$K_{3}=(\sqrt{-195}, \sqrt{5})$ & $C_{3}^{z} \quad(z=0,1, \ldots 7)$ & $c_{3} \sim 1, c_{1} \sim c_{2} \chi 1$ \\
$\left(\frac{-39}{\mathfrak{p}_{5}}\right)_{k}=\left(\frac{-39}{5}\right)=+1$ & & \\
\hline
\end{tabular}

Die Angaben dieser Tabelle folgen zum T'eil aus den allgemeinen Untersuchungen des $\& 3$, zum Teil sollen sie im folgenden bewiesen werden.

Da im Körper $K_{2}$ neben 5 noch die singuläre Primärzahl $-1+2 \sqrt{-3}=(\sqrt{65}, 1+2 \sqrt{-3})^{2}$ existiert, kann seine Klassengruppe nicht zyklisch sein; es muß daher der Klassenkörper von $k(\sqrt{-195})$ den Typus $O$ repräsentieren. Daß dann die Klassengruppe von $K_{3}$ zyklisch sein muß, folgt daraus, daß in ihm eine Klasse mit dem Exponenten 4 existiert; dab die Klassenzahl von $K_{3}$ gleich 8 ist, mub noch bewiesen werden.

Nach den bisherigen Ausführungen kann diese Klassenzahl nur 4 oder 8 sein. Wäre sie gleich 4 , so würde der Klassenkörper $K^{2} k$ von $K_{1}$ oder $K_{2}$ oder $K_{3}$ in bezug auf $K_{1}$ und $K_{2}$ den Typus $A$ repräsentieren; Primideale aus $c_{1}$ würden dann in $K_{1}$ in zwei verschiedene Primideale zerfallen und jedes von diesen würde in $K^{2} k$ wieder in zwei verschiedene Primideale zerfallen, so daß also die Primideale ans $c_{1}$ in $K^{2} \hbar$ in vier versehiedene Primideale zerfallen würden; dasselbe würde von den Primidealen aus $c_{2}$ gelten. Dann müßte aber in $K_{3}$ :

$$
c_{1} \sim c_{2} \propto 1
$$

sein, was unmöglich ist, wie wir zeigen wollen. 
Da der Körper $K_{3}$ unverzweigt vom Relativgrad 2 in bezug auf $k$ ist, gilt für ihn unter Benützung der üblichen Bezeichnungen: ${ }^{1}$ )

$$
v^{*}=m^{\prime}-e_{1}+1 \text {. }
$$

Nun ist in unserem Falle $m^{\prime}=1$ und wegen

$$
-1=(2+\sqrt{5})(2-\sqrt{5})
$$

auch $v^{*}==1$. Es folgt also $e_{1}=1, d$. h. es kann nur ein unabhängiger Klassenverband aus $k$ in $K_{3}$ in die Hauptklasse übergehen. Wegen $\mathfrak{p}_{5} \sim 1$ in $K_{3}$ mufo deshalb gelten:

$$
c_{3} \sim 1, c_{1} \sim c_{2} \sim 1 \text { in } K_{3} \text {. }
$$

Gleichzeitig folgt, daß die Klassenzahl von $K_{3}$ gleich 8 ist.

Unsere Körper liefern uns auch ein Beispiel für den Typus $B$. Im Klassenkörper $K^{2} k$ des Klassenkörpers $K k$ zerfallen die Primideale aus $c_{1}$ und $c_{2}$ in je vier verschiedene Primfaktoren, während die Primideale aus $c_{3}$ nur in zwei Faktoren zerfallen. In $K^{2} k$ zerfallen daher alle Primideale aus $K_{1}$, die nicht in der Hauptklasse liegen, in zwei verschiedene Primfaktoren, so daß der Klassenkörper von $K_{1}$ (oder $K_{2}$ ) den Typus $B$ repräsentiert.

Wir haben daher folgende Zusammenstellung:

\begin{tabular}{|c|c|c|c|}
\hline K ö r p e r & Klassenzahl & $\begin{array}{c}\text { Klassenzahl des } \\
\text { Klassenkörpers }\end{array}$ & Typus \\
\hline$(\sqrt{-2})$ & 4 & 1 & $A$ \\
\hline$(\sqrt{-195}, \sqrt{13})$ & 4 & 2 & $B$ \\
\hline$(\sqrt{-195})$ & 4 & 4 & $C$ \\
\hline
\end{tabular}

\$ 6.

Um den Fall zu erledigen, daß die Klassenzahl des Grundkörpers $k$ gleich $l^{2}$ ist und seine Klassengruppe:

$$
c_{1}^{x_{1}} c_{2}^{x_{2}} \quad(x=0,1, \ldots l-1),
$$

kann man ganz analog wie in $\S 1$ verfahren, so daß ich mich kurz fassen kann. Zur Untergruppe $c_{1}^{x_{1}}$ der Klassengruppe von $k$ gehört ein unverzweigter Körper $K_{1}$ vom Relativgrad $l$ in bezug anf $k$, dessen Klassensystem in der Gestalt:

$$
C_{1}^{F(S)}
$$

1) $\mathbf{P h}$. Furtwängler, Öber die Konstr. des Klassenkörpers nsw., Gött. Nachr., 1903, p. 295, Satz 8. 
darstellbar ist, wo $C_{1}$ eine Klasse aus $K_{1}$ bedeutet, deren Relativnorm in $k$ in $c_{1}$ fällt und $S$ eine erzeugende Substitution der Relativgruppe des Körpers $K_{1}$ bezüglich $k$ ist. Enthält $K_{1}$ eine ambige Klasse, so wird bereits in $K_{1}$ :

$$
c_{1} \sim c_{2} \sim 1 \text {. }
$$

Enthält $K_{1}$ keine ambige Klasse, so muß es doch in $K_{1}$ eine Klasse $C^{\prime}$ geben, die in $K_{1}$ nicht Hauptklasse ist, aber im Klassenkörper $K k_{i}$ von $k$ in die Hauptklasse übergeht. Mit $C^{\prime}$ liegen dann auch alle Klassen $C_{i}=C^{(S-1)^{i}}$ in $K k$ in der Hauptklasse. Da die symbolische $(S-1)^{l_{\text {te }}}$ Potenz auch wirkliche $l^{\text {te }}$ Potenz einer Klasse ist, muß es dann einen' Index $n$ geben, so dab:

$$
C_{n+1}^{q} \sim 1, \quad C_{n}^{q} \nsim 1 \text { in } K_{1},
$$

wo $q$ einen zu. $l$ primen Exponenten bedeutet. Es ist dann

$$
C_{n}^{q(S-1)} \sim 1
$$

und da keine ambige Klasse in $K_{1}$ existiert, muß

$$
C_{n}^{q} \sim c \propto 1
$$

gelten, wo $c$ eine Klasse aus $k$ bedeutet, die in $K_{1}$ nicht in der Hauptklasse liegt, aber in $K k$ in die Hauptklasse übergeht. Da bei dem Übergang von $k$ nach $K_{1}$ stets auch ein Klassenverband in die Hauptklasse übergeht, so gehen also in jedem Falle alle Klassen aus $k$ im Klàssenkörper $K k$ in die Hauptklasse über.

Für die vorstehenden Betrachtungen ist es gleichguiltig, ob der Grundkörper $k$ eine primitive $~^{\text {te }}$ Einheitswurzel enthält oder nicht, wenn auch die benützten Überlegungen zum Teil nur für den ersten Fall in meinen fruheren Arbeiten durchgeführt sind; ihre Übertragung auf den zweiten Fall bietet keine Schwierigkeiten. 